\title{
Effect of a lifestyle intervention during pregnancy-findings from the Finnish gestational diabetes prevention trial (RADIEL)
}

\section{Rönö, Kristiina}

2018-09

Rönö , K , Grotenfelt , N E , Klemetti , M M , Stach-Lempinen , B , Huvinen , E , Meinilä , J , Valkama, A, Tiitinen , A, Roine , R P , Pöyhönen-Alho, M , Andersson , S, Laivuori , H , Kautiainen , H , Eriksson , J G \& Koivusalo , S B 2018 , ' Effect of a lifestyle intervention during pregnancy-findings from the Finnish gestational diabetes prevention trial (RADIEL) ' , Journal of Perinatology , vol. 38 , no. 9 , pp. 1157-1164 . https://doi.org/10.1038/s41372-018-0178-8

http://hdl.handle.net/10138/322356

https://doi.org/10.1038/s41372-018-0178-8

acceptedVersion

Downloaded from Helda, University of Helsinki institutional repository.

This is an electronic reprint of the original article.

This reprint may differ from the original in pagination and typographic detail.

Please cite the original version. 
This is a post-peer-review, pre-copyedited version of an article published in Journal of Perinatology

The final authenticated version is available online at: https://doi.org/10.1038/s41372-018-0178-8

\section{Effect of a lifestyle intervention during pregnancy - findings from the Finnish gestational diabetes prevention trial (RADIEL)}

Kristiina Rönö, $\mathrm{MD}^{1}$; Nora Elisabeth Grotenfelt, $\mathrm{MD}^{2}$; Miira Marjuska Klemetti, MD $\mathrm{PhD}^{1,3,9}$; Beata Stach-Lempinen, $\mathrm{MD} \mathrm{PhD}^{3}$; Emilia Huvinen, $\mathrm{MD}^{1}$; Jelena Meinilä, MSc

$\mathrm{PhD}^{2}$; Anita Valkama, $\mathrm{MSc}^{2,4}$; Aila Tiitinen, $\operatorname{Prof}^{1}$, Risto Paavo Roine, Prof ${ }^{5,6,7}$; Maritta Pöyhönen-Alho MD PhD ${ }^{1}$; Sture Andersson, $\operatorname{Prof}^{8}$; Hannele Laivuori, Associate prof ${ }^{9,10,11,12}$; Hannu Kautiainen, biostatistician ${ }^{2,13}$; Johan Gunnar Eriksson, Prof ${ }^{2,4,14}$; Saila Birgitta Koivusalo, Adjunct prof ${ }^{1}$

1. Obstetrics and Gynecology, University of Helsinki and Helsinki University Hospital, Helsinki, Finland

2. Unit of General Practice and Primary Health Care, University of Helsinki and Helsinki University Hospital, Helsinki, Finland

3. Obstetrics and Gynecology, South Karelia Central Hospital, Lappeenranta, Finland

4. Folkhälsan Research Center, Helsinki, Finland

5. Department of Health and Social Management, Research Centre for Comparative Effectiveness and Patient Safety, University of Eastern Finland, Kuopio, Finland

6. Kuopio University Hospital, Kuopio, Finland

7. Administration, University of Helsinki and Helsinki University Hospital, Helsinki, Finland

8. Children's Hospital, University of Helsinki and Helsinki University Hospital, Helsinki, Finland

9. Medical and Clinical Genetics, University of Helsinki and Helsinki University Hospital, Helsinki, Finland

10. Institute for Molecular Medicine Finland, Helsinki Institute of Life Science, Helsinki, Finland 
11. Faculty of Medicine and Life Sciences, University of Tampere, Tampere, Finland

12. Department of Obstetrics and Gynecology, Tampere University Hospital, Tampere, Finland

13. General Practice and Primary Health Care, University of Eastern Finland, Kuopio, Finland

14. Department of Chronic Disease Prevention, National Institute for Health and Welfare, Helsinki, Finland

\section{Correspondence:}

Kristiina Rönö, Women's Hospital, P.O. Box 140, FIN-00029 HUS, Finland

Email. kristiina.rono@ helsinki.fi, Tel.+358 50427118

Running title: Early gestational diabetes common in women at risk

Funding: The RADIEL study is funded by the Jalmari and Rauha Ahokas Foundation, the Finnish Foundation for Cardiovascular Research, State funding for university-level health research of Helsinki University Hospital and South Karelia Central Hospital, Samfundet Folkhälsan, the Finnish Diabetes Research Foundation, the State Provincial Office of Southern Finland, The Vyborg Tuberculosis Foundation, and the Social Insurance Institution of Finland. In addition, The Finnish Medical Foundation, the Finnish Diabetes Research Foundation, the Finnish Cultural Foundation, the Juho Vainio Foundation, and the Helsinki University Doctoral School in Health Sciences supported the PhD study of the first author. The Academy of Finland supported JGE [grant No. 129369, 129907, 135072, 129255, and 126775]. The research leading to these results has received funding from the European Commission within the 7th Framework Programme and EU H2020-PHC-2014-DynaHealth grant no. 633595. None of the funding bodies had any role in the design of the study, collection and analysis of data or decision to publish. 


\begin{abstract}
Objective

To assess the effect of lifestyle counseling on perinatal outcomes among women at high risk for gestational diabetes.

Study Design

492 women with obesity and/or prior gestational diabetes were allocated to intervention (four sessions of lifestyle counseling, $n=249)$ or usual care $(n=243)$ before 20 weeks' gestation.

Result

Lifestyle indicators, gestational weight gain or obstetric and perinatal outcomes did not differ between the two groups. An oral glucose tolerance test in the first half of pregnancy was pathological in $37.7 \%(n=87 / 144)$ of intervention and $36.5 \%(n=72 / 197)$ of control group women $(\mathrm{p}=0.81)$. The total incidence of gestational diabetes diagnosed in the first or second half of pregnancy was $44.8 \%(107 / 239)$ in the intervention and $48.1 \%(111 / 231)$ in the control group $(\mathrm{p}=0.48)$.

\section{Conclusion}

The high prevalence of impaired glucose metabolism was observed already in early pregnancy, which may have contributed to the lack of effect of the intervention.

Trial registration ClinicalTrials.gov IDr: NCT01698385 


\section{Introduction}

Hyperglycemia during pregnancy is associated with adverse maternal and neonatal outcomes. ${ }^{1-3}$ The maternal ramifications extend beyond pregnancy as gestational diabetes mellitus (GDM) predicts the onset of type 2 diabetes (T2D). ${ }^{4}$ In addition, the offspring of women with hyperglycemia during pregnancy are at increased risk for obesity, ${ }^{3}$ impaired glucose regulation, and higher blood pressure levels. ${ }^{5}$ Higher risk for adiposity ${ }^{5}$ among girls further enhances the vicious cycle of diabetes and obesity over generations.

Several studies aiming at prevention of GDM or related adverse outcomes have been undertaken. These studies have included either diet or physical activity interventions, or both. In terms of GDM prevention, the results have been inconsistent. ${ }^{6-16}$ A recent meta-analysis concluded, however, that interventions initiated in early pregnancy have the potential to prevent GDM. ${ }^{17}$ The efficacy of intervention trials is usually highest among participants at high risk, as seen for example in the prevention of T2D. ${ }^{18,19}$ The largest GDM trials have, however, included also women at low or medium risk. Surprisingly few studies have assessed the participants' glucose tolerance at enrollment. ${ }^{7,14}$

While having no significant effect on the incidence of GDM, many intervention trials have been effective in improving lifestyle, ${ }^{10,12}$ in reducing the incidence of large for gestational age (LGA) newborns or macrosomia, ${ }^{7,11,15}$ or in limiting gestational weight gain $(\mathrm{GWG}) .^{8-}$ $10,12,14,16$

The Finnish gestational diabetes prevention trial (RADIEL) was designed to evaluate the efficacy of a combined diet and physical activity intervention, applicable to a primary health 
care setting, in the prevention of GDM and obesity-related maternal and neonatal complications among high-risk women (i.e., obese and/or with a history of GDM). RADIEL was a multi-center randomized controlled trial targeting women planning pregnancy within one year and pregnant women before 20 weeks of gestation. ${ }^{20} \mathrm{~A} 2-\mathrm{h} 75 \mathrm{~g}$ oral glucose tolerance test (OGTT) was undertaken before 20 weeks of gestation. Among a subgroup of women recruited in early pregnancy, with normal glucose tolerance at inclusion, the risk of GDM was reduced by $36 \%$ by the intervention. ${ }^{21}$ The aim of this study was to assess the effect of lifestyle counseling on diet, physical activity as well as maternal and perinatal outcomes within the whole study population recruited in early pregnancy.

\section{Methods}

Study design and setting

The RADIEL trial was conducted in Finland between February 2008 and January 2014 at four study sites: the three maternity hospitals of the Helsinki metropolitan area (Department of Obstetrics and Gynecology, Helsinki University Hospital (HUH), Helsinki; Kätilöopisto Maternity Hospital, Helsinki; Department of Obstetrics and Gynecology, Jorvi Hospital, Espoo) and the South-Karelia Central Hospital (SKCH) in Lappeenranta. This study presents the results from the part of the RADIEL trial in which the intervention was initiated during pregnancy before 20 weeks of gestation, leaving out the participants who started the intervention while planning pregnancy. In our previous publication, ${ }^{21}$ we presented the results of the intervention among women with normal glucose tolerance in early pregnancy. In this study, we analyzed the impact of the lifestyle intervention among all women enrolled in early 
pregnancy, regardless of their glucose tolerance at inclusion. The design and methods of the intervention have been published in detail previously, and are only summarized here. ${ }^{20,21}$

Women $\geq 18$ years of age, with a prepregnancy body mass index $(\mathrm{BMI}) \geq 30 \mathrm{~kg} / \mathrm{m}^{2}$ or a history of GDM in a previous pregnancy were eligible for the study. Women with diabetes diagnosed before pregnancy, medication that influences glucose metabolism (such as oral corticosteroids or metformin), multiple pregnancy, physical disability that prevents regular exercise, current substance abuse, severe psychiatric disorder (i.e. psychotic disorders, schizophrenia, or schizoaffective disorders), or inadequate Finnish language skills (insufficient for receiving counseling and for completing questionnaires) were excluded.

Participants were mainly recruited in connection with the first-trimester ultrasound screening or by personal invitation letters based on data on prior GDM from the maternity hospital registry. All participants signed an informed consent and were allowed to discontinue at any time during the study. The study complied with the Declaration of Helsinki and was approved by the Ethics Committees of HUH (14 September 2006, Dnro 300/E9/06) and SKCH (11 September 2008, Dnro M06/08). The RADIEL study was registered at ClinicalTrials.gov (IDr: NCT01698385).

\section{Sample size and randomization}

We calculated the sample size for the whole RADIEL trial, including both women planning pregnancy and women already pregnant, assuming a GDM incidence of $30 \%$ in the control arm and of $20 \%$ in the intervention arm, using a two-sided significance level of 0.05 , and a power of $80 \%$. We expected a dropout rate of $30 \%$. A sample size of at least 1000 women for 
the whole trial was estimated to be sufficient. The randomization was stratified by the time of initiation of the intervention (i.e., prepregnancy or early pregnancy), study site, and risk factor (BMI $\geq 30 \mathrm{~kg} / \mathrm{m}^{2}$, prior diet-treated GDM, and prior GDM treated with medication). This manuscript reports results of the subgroup of participants recruited in early pregnancy leaving out the participants who started the intervention already while planning pregnancy.

\section{Intervention}

The participants in both study arms visited the study nurse three times during pregnancy in addition to receiving usual antenatal care. The study visits took place on average at 13,23 , and 35 weeks of gestation. At each study visit, participants in both study arms filled in questionnaires and underwent a physical examination including anthropometric and blood pressure measurements as previously described. ${ }^{20}$

During the study visits, participants in the intervention arm received structured but individually modified dietary and physical activity counseling from the study nurses. In addition, the participants in the intervention arm attended a group nutrition counseling session given by a dietitian after the first study visit.

Dietary counseling in the intervention group was based on Nordic Nutrition Recommendations at the time of the study. ${ }^{22}$ The counseling focused on food intake: encouraging the use of vegetables, fruits and berries, high fiber whole grain products, low-fat dairy products, vegetable fats high in unsaturated fatty acids, fish and low-fat meat products, and lower intake of sugar- and saturated fatty acid-rich foods. For the intervention arm, the recommendation for physical activity was a minimum of 150 minutes of moderate intensity 
physical activity per week. ${ }^{23}$ Women with a prepregnancy BMI $\geq 30 \mathrm{~kg} / \mathrm{m}^{2}$ were advised to avoid weight gain during the first two trimesters of pregnancy.

The participants in the control group had an equal number of visits to the study nurse as the participants in the intervention arm. They received information leaflets on healthy diet and exercise at enrollment, similar to those provided in public antenatal care.

\section{Usual antenatal care in Finland}

The public health care system in Finland offers all pregnant women antenatal health care on a regular basis in municipal maternity clinics at primary health care centers. All participants in our study, both in the intervention and in the control arm, received this routine care, in addition to the study visits. At the time of the study, the usual antenatal follow-up in primary care included 10 to 15 visits with a nurse and two to three physician's examinations. Following the national GDM guidelines, the women diagnosed with GDM at any time point during pregnancy (including early pregnancy) received routine care, including diet and physical activity recommendations, and self-monitoring of blood glucose, in the municipal

maternity clinics. ${ }^{24}$ In case of repeated fasting capillary glucose of $\geq 5.5 \mathrm{mmol} / \mathrm{L}$ or a onehour postprandial value of $\geq 7.8 \mathrm{mmol} / \mathrm{L}$, the maternity clinic referred the woman to a maternity hospital for further counseling by a diabetes nurse and to an obstetrician for assessment regarding need of medication.

\section{Outcomes}


The primary outcome of the RADIEL trial was the total incidence of GDM during pregnancy (including incidence in early pregnancy i.e. at less than 20 weeks of gestation). The assessed pre-specified secondary outcomes were achievement of dietary and physical activity goals, incidence of hypertensive disorders of pregnancy; i.e. gestational hypertension, preeclampsia, and chronic hypertension; gestational weight gain $(\mathrm{GWG})$; mode of delivery; perinatal outcome, i.e. birth weight of the offspring and rate of large for gestational age (LGA) newborns. ${ }^{20}$

To calculate the incidence of GDM we used the criteria recommended by the Finnish Current Care Guidelines where GDM is defined as one or more pathological glucose values in a 75-g 2h OGTT during pregnancy. ${ }^{24}$ The diagnostic thresholds were based on those recommended by the American Diabetes Association in 2008: fasting plasma glucose $\geq 5.3 \mathrm{mmol} / \mathrm{L}$, one hour value $\geq 10.0 \mathrm{mmol} / \mathrm{L}$ and two-hour value $\geq 8.6 \mathrm{mmol} / \mathrm{L} .{ }^{25}$ Following the Finnish Current Care Guidelines for GDM diagnosis, the participants underwent an OGTT at around 12 to 16 weeks of gestation, and, if normal, the OGTT was repeated around 24 to 28 weeks of gestation unless insulin or metformin treatment was initiated earlier. ${ }^{24}$

In order to measure the general adherence to the recommended diet, our research group developed a Healthy Food Intake Index (HFII, with the maximum points of 17) ), which has later been validated, based on the food frequency questionnaire designed for the RADIEL study. ${ }^{26}$ Physical activity was assessed with self-reported weekly duration (minutes) of leisure time physical activity (LTPA) that makes the participant at least slightly out of breath and sweating. In addition, participants filled out three-day food diaries and one-week physical activity diaries at baseline and third trimester. We used Compendium of Physical Activities 
codes for each activity and corresponding MET values when recording the physical activity diaries. $^{27}$

The definition of preeclampsia was a systolic blood pressure $\geq 140 \mathrm{mmHg}$ and/or a diastolic blood pressure $\geq 90 \mathrm{mmHg}$ appearing for the first time after 20 weeks of gestation together with proteinuria $\geq 0.3 \mathrm{~g} / 24 \mathrm{hr} .^{28}$ We defined hypertension as chronic if similar blood pressure levels occurred before 20 weeks of gestation, or the participant used antihypertensive medication. The definition of gestational hypertension was new onset hypertension after 20 weeks of gestation without proteinuria. We used population-specific Finnish references for birth weight at the time of the trial to evaluate the birth size of the offspring and defined LGA as birth weight $>2.0$ standard deviation (SD). ${ }^{29}$ The GWG of the participant was calculated by subtracting the self-reported prepregnancy weight, collected from the antenatal clinic records, from the weight measured at the third-trimester study visit. If the participant did not attend the third trimester study visit, the weight was collected from maternity records when available. We collected data on maternal and neonatal outcomes from hospital records. Study physicians confirmed the maternal and neonatal diagnoses.

\section{Statistical analyses}

Means with SD or medians with interquartile range (IQR) were used to describe continuous data. For nominal data counts with percentages $(\%)$ were used. Student's t-test, permutation test, Mann-Whitney test, and chi-square or Fisher's exact test were used in comparisons between groups. A logistic regression model was applied when using adjusted models. The analyses were performed using STATA software (version 14.1), StataCorp, LP, Texas, USA. 


\section{Results}

We recruited 540 women at high risk for GDM to the study in the first half of pregnancy (mean 13.1 [SD 1.9] weeks of gestation). The number of women included in the analyses was 470, as 70 women did not provide informed consent, did not meet inclusion criteria, lacked OGTT data, or experienced pregnancy loss before OGTT (Figure 1). Of these 470 participants, $3.3 \%(n=8)$ in the intervention group and $14.7 \%(n=34)$ in the control group did not participate in an OGTT during the first half of pregnancy. Four women (three in the intervention group and one in the control group) had an early pregnancy loss after a pathologic OGTT, and an additional two women (one in both groups) were missing data on delivery (due to changing residence during the trial) and were included only in the analyses of GDM incidence. Third-trimester weight data were not available for 12 women in the intervention group and five women in the control group. Of the women in intervention group $63.2 \%(n=151)$ and in the control group $59.3 \%(n=137)$ returned both food diaries. The respective rates for returning both physical activity diaries were $44.8 \%(\mathrm{n}=107)$ and $46.3 \%$ $(n=107)$, respectively.

Demographic and clinical characteristics of the randomized women are presented in Table 1. Of the 470 participants, $35.5 \%(n=167)$ had prior GDM treated with diet (dGDM), $5.3 \%$ $(n=25)$ had prior GDM treated with medication $(\mathrm{mGDM})$, and $59.2 \%(\mathrm{n}=278)$ were either nulliparous or had no history of GDM, with obesity being the only criteria for inclusion. Of the 192 participants with prior GDM, 38.5\% $(n=74)$ were obese. The women recruited due to obesity only were younger (31.7 years [SD 5.0], compared with 33.4 years [SD 4.5] in dGDM and 33.3 years [SD 4.3] in mGDM), had fewer deliveries (median 0 [IQR 0 to 1], compared with 1 [IQR 1 to 2] in dGDM and 2 [IQR 1 to 2] in mGDM), had lower fasting 
plasma glucose at inclusion $(5.00 \mathrm{mmol} / \mathrm{L}$ [SD 0.41], compared with $5.12 \mathrm{mmol} / \mathrm{L}$ [SD 0.38] in dGDM and $5.26 \mathrm{mmol} / \mathrm{L}$ [SD 0.52] in mGDM), and had higher BMI (34.5 kg/m² [SD 3.9], compared with $28.7 \mathrm{~kg} / \mathrm{m}^{2}$ [SD 6.3] in dGDM and $28.6 \mathrm{~kg} / \mathrm{m}^{2}$ [SD 5.7] in mGDM) compared with women recruited due to prior GDM $(\mathrm{p}<0.001)$.

The total incidence of GDM during pregnancy was $44.8 \%(n=107,95 \%$ CI $38.4 \%-51.3 \%)$ in the intervention group and $48.1 \%(\mathrm{n}=111,95 \%$ CI $41.5 \%-54.7 \%)$ in the control group $(\mathrm{p}=$ 0.48 , unadjusted; $\mathrm{P}=0.44$, after adjustment for age, prepregnancy BMI, previous GDM status, and parity). The crude OR for GDM was 0.88 (95\% CI 0.61 to 1.26 ) in the intervention group. The incidence of early GDM in the intervention group was $37.7 \%(n=87)$ and in the control group $36.5 \%(n=72)$ among the 428 women who attended the early pregnancy OGTT $(\mathrm{p}=0.81)$.

The dietary HFII score improvement from enrollment to the third-trimester visit was 0.8 points (95\% CI: 0.5 to 1.2$)$ in the intervention group and 0.4 points (95\% CI: 0.0 to 0.8 ) in the control group $(\mathrm{p}=0.10)$. The women in the intervention arm increased their total energy intake by $231 \mathrm{~kJ}$ per day (SD 1703) and the women in the control arm by $104 \mathrm{~kJ}$ per day (SD 1979) between baseline and the third trimester visit $(\mathrm{p}=0.56)$. No differences between the groups were detected in the intakes (percent of total energy intake) of fat, protein, carbohydrate, or dietary fiber (data not shown).

Women in the intervention group decreased their median weekly LTPA from the baseline to the third trimester visit by $15 \mathrm{~min}(95 \% \mathrm{CI}-5$ to $-30 \mathrm{~min})$, while the women in the control group decreased theirs by $22 \min (95 \% \mathrm{CI}:-10$ to -32$)(\mathrm{p}=0.65)$. In the third trimester, 
$15.3 \%(n=29 / 190)$ of the women in the intervention group and $13.7 \%(n=23 / 168)$ in the control group met the physical activity goal of at least 150 minutes per week $(\mathrm{p}=0.53)$. The women in the intervention arm decreased their weekly MET minutes by 53 (SD 949) and the women in the control arm by 182 (SD 929) between baseline and third-trimester visit $(\mathrm{p}=0.32)$.

No differences were observed between the intervention and control groups regarding assessed obstetric or perinatal outcomes (Table 2).

\section{Discussion}

Among all the high-risk women randomized in the RADIEL trial in early pregnancy, including women with early GDM, we detected no effect of the lifestyle intervention on dietary intake or physical activity. Consequently, related parameters such as gestational weight gain and the total incidence of GDM were not affected. Furthermore, there were no differences between the groups regarding obstetric or perinatal outcomes, e.g., hypertensive disorders of pregnancy, birthweight, and rate of LGA. These results differ from our previous findings among a subgroup of participants with normal glucose tolerance in early pregnancy. $^{21}$

There were no statistically significant differences between the intervention group and the control group regarding changes in dietary score and total energy intake. This is in contrast to some previous interventions, which have reported improved dietary quality or diminished total energy intake. . $^{70,12,14,30}$ 
As expected, the weekly duration of physical activity decreased throughout pregnancy. However, the change in duration of physical activity was similar in both groups, indicating a null effect of the intervention on physical activity. In addition, the majority of the participants did not reach the target of 150 minutes of physical activity per week. This is in contrast to some previous intervention trials which have reported higher physical activity levels in the intervention group compared with the control group..$^{12,14,16}$

Some previous lifestyle intervention trials combining diet and physical activity have successfully reduced GWG. ${ }^{9,10,12,30}$ For instance, the combined healthy eating and physical activity intervention in the DALI study succeeded in reducing GWG compared to isolated dietary or physical counseling. ${ }^{14}$ Furthermore, the Finnish NELLI study succeeded in reducing both birthweight and the rate of LGA newborns, with no effect on the rate of macrosomia. $^{7}$

In some trials, physical activity interventions alone have been effective. Three trials, consisting of supervised exercise sessions three times a week have managed to limit GWG and reduce the incidence of GDM..$^{13,15,16}$ In addition, one of these was successful in reducing the rates of pregnancy-induced hypertension, preeclampsia, and macrosomia, ${ }^{15}$ while another succeeded in reducing birthweight. ${ }^{16}$ In all of these studies, the intervention group was significantly more physically active compared with the control group. We believe that our physical activity counseling was of notably lower intensity and also less intense in general compared to trials with a positive effect on obstetric or perinatal outcomes. ${ }^{13,15,16}$

As shown previously, in a subgroup consisting of women with normal OGTT in early pregnancy, we detected improvements in lifestyle and coincidentally a reduced the risk of 
GDM by $36 \% .{ }^{21,31}$ However, in the present high-risk cohort we detected no effect of the same intervention. There are several possible reasons for this discrepancy. A significant proportion (37\%) of the women in the present high-risk population fulfilled the diagnostic criteria for GDM already in the first half of pregnancy. The fact that all women with a diagnosis of early GDM in the intervention and control groups received routine counseling and treatment within the Finnish health care system may have attenuated the differences in effect of the RADIEL intervention on lifestyle, obstetric, and perinatal outcomes between the intervention and control groups.

Considering the high standard of usual care, including recommendations on diet and physical activity for all women and treatment for women with both early and later GDM, we hypothesize that the intensity of the given intervention in the RADIEL trial (the maximum of four study visits, including the group session with a dietitian) was probably not high enough to provide additional benefit for participants in the intervention group.

Insulin resistance increases as pregnancy progresses and lifestyle interventions usually aim at preventing GDM mainly through attempts to reduce insulin resistance which also has a positive impact on insulin secretion. Considering the heterogeneous nature of GDM, it is likely that the potential of any lifestyle intervention to prevent GDM is variable, depending on the type and degree of impairment in glucose metabolism in the study population. In our study, a high proportion of the participants had a pathological OGTT already in early pregnancy, potentially suggesting a more severe form of derangement in glucose metabolism. It is possible that the predisposition to develop early GDM was strong in our study population, due to both genetic and/or environmental factors, and thus a mild-to-moderate 
intensity lifestyle intervention initiated during pregnancy was not sufficient to have a significant impact on glucose metabolism or other obstetric or perinatal outcomes.

In order to be able to assess the effect of a lifestyle intervention during pregnancy on GDM risk, glucose tolerance must be assessed before study inclusion. To our knowledge, most lifestyle intervention trials aimed at prevention of GDM or obesity-related outcomes have not tested the participants for GDM in early pregnancy. Exceptions are the NELLI study, in which about $28 \%$ of the assessed women were excluded due to pathologic OGTT results at inclusion, ${ }^{7}$ and the DALI study, in which the corresponding rate was $27 \% .^{14}$

Many prevention trials, including the RADIEL trial, have utilized national GDM criteria. The use of variable diagnostic thresholds and testing times for the diagnosis of GDM further complicates the comparison of results from different studies. In addition, differences in national treatment guidelines for GDM might lead to substantial variations in the impact of additional interventions compared to routine care.

The strength of this study is the characterization of the participants at baseline, including assessment of early pregnancy glucose metabolism. This adds valuable information for future studies on timing of prevention of GDM and its adverse effects. The small sample size is a limitation as it does not allow detection of differences in relatively infrequent adverse obstetric and perinatal outcomes. Furthermore, our sample size and the fact that not all participants attended the early pregnancy OGTT prevent a more detailed exploration of the early GDM subgroup. In addition, the applied diet and physical activity measurement methods might have been too crude to detect small changes. Physical activity was self- 
reported and not based on e.g., accelerometer. The return rates of food and physical activity diaries were low, and we lack diary data concerning the second trimester.

One might argue that the duration of lifestyle interventions during pregnancy is too short to prevent GDM and related outcomes and that interventions should be initiated already in the pre-pregnancy period. We stress the need for future studies exploring the efficacy of GDM prevention interventions initiated in the pre-pregnancy period.

In conclusion, many of the high-risk women developed GDM already in early pregnancy. The combined dietary and physical activity counseling initiated in early pregnancy in the RADIEL trial in women at high risk of GDM did not affect dietary intake, physical activity, or obstetric and perinatal outcomes. These results differ from our previous sub-study including only women with normal glucose tolerance in early pregnancy.

\section{Acknowledgements}

The authors acknowledge the contribution of the study nurses Hanna Oksa, Kirsi Arponen, Päivi Saksa, Maaret Ahola, Pirkko Haapanen, Karoliina Himanen, and Eija Kortelainen, dietitians Riitta Kivelä, xxx, and Johanna Metsälä, as well as the women participating in the study.

\section{Conflict of interest.}

The authors declare no conflict of interest.

\section{Authorship}


KR and NEG wrote the first draft of the manuscript, participated in the implementation of the study, the statistical analyses and interpretation of the results. In addition, KR prepared the database for statistical analyses. SBK initiated the RADIEL study and participated in the design, planning, and implementation of the study in collaboration with MMK, BSL, AT, MPA, SA, and HL. EH, RPR, and JGE participated in the implementation of the study. SBK coordinated the study in Helsinki and BSL in Lappeenranta. AV and JM participated in the implementation and statistical analyses and interpretation of the nutritional aspects of the study. HK supervised the statistical analyses and participated in the interpretation of the results. All authors contributed to the revision of the manuscript and approved the final version. KR has had full access to the data in the study and final responsibility for the decision to submit for publication. 


\section{References}

1 Jolly MC, Sebire NJ, Harris JP, Regan L, Robinson S. Risk factors for macrosomia and its clinical consequences: a study of 350,311 pregnancies. European Journal of Obstetrics \& Gynecology and Reproductive Biology 2003; 111: 9-14.

2 Farrar D, Simmonds M, Bryant M, Sheldon TA, Tuffnell D, Golder S et al. Hyperglycaemia and risk of adverse perinatal outcomes: systematic review and metaanalysis. BMJ 2016; 354: i4694.

3 Zhu Y, Olsen SF, Mendola P, Yeung EH, Vaag A, Bowers K et al. Growth and obesity through the first $7 \mathrm{y}$ of life in association with levels of maternal glycemia during pregnancy: a prospective cohort study. Am J Clin Nutr 2016; 103: 794-800.

4 Bellamy L, Casas J-P, Hingorani AD, Williams D. Type 2 diabetes mellitus after gestational diabetes: a systematic review and meta-analysis. Lancet 2009; 373: $1773-$ 1779.

5 Tam WH, Ma RCW, Ozaki R, Li AM, Chan MHM, Yuen LY et al. In Utero Exposure to Maternal Hyperglycemia Increases Childhood Cardiometabolic Risk in Offspring. Diabetes Care 2017; 40: 679-686.

6 Quinlivan JA, Lam LT, Fisher J. A randomised trial of a four-step multidisciplinary approach to the antenatal care of obese pregnant women. Aust N Z J Obstet Gynaecol 2011; 51: 141-146.

7 Luoto R, Kinnunen TI, Aittasalo M, Kolu P, Raitanen J, Ojala K et al. Primary prevention of gestational diabetes mellitus and large-for-gestational-age newborns by lifestyle counseling: a cluster-randomized controlled trial. PLoS Med 2011; 8: e1001036.

8 Vinter CA, Jensen DM, Ovesen P, Beck-Nielsen H, Jørgensen JS. The LiP (Lifestyle in Pregnancy) study: a randomized controlled trial of lifestyle intervention in 360 obese pregnant women. Diabetes Care 2011; 34: 2502-2507.

9 Renault KM, Nørgaard K, Nilas L, Carlsen EM, Cortes D, Pryds O et al. The Treatment of Obese Pregnant Women (TOP) study: a randomized controlled trial of the effect of physical activity intervention assessed by pedometer with or without dietary intervention in obese pregnant women. Am J Obstet Gynecol 2014; 210: 134.e1-9.

10 Hui AL, Back L, Ludwig S, Gardiner P, Sevenhuysen G, Dean HJ et al. Effects of lifestyle intervention on dietary intake, physical activity level, and gestational weight gain in pregnant women with different pre-pregnancy Body Mass Index in a randomized control trial. BMC Pregnancy Childbirth 2014; 14: 331.

11 Dodd JM, Turnbull D, McPhee AJ, Deussen AR, Grivell RM, Yelland LN et al. Antenatal lifestyle advice for women who are overweight or obese: LIMIT randomised trial. BMJ 2014; 348: g1285.

12 Poston L, Bell R, Croker H, Flynn AC, Godfrey KM, Goff L et al. Effect of a behavioural intervention in obese pregnant women (the UPBEAT study): a multicentre, 
randomised controlled trial. Lancet Diabetes Endocrinol 2015; 3: 767-777.

13 Cordero Y, Mottola MF, Vargas J, Blanco M, Barakat R. Exercise Is Associated with a Reduction in Gestational Diabetes Mellitus. Medicine \& Science in Sports \& Exercise 2015; 47: 1328-1333.

14 Simmons D, Devlieger R, van Assche A, Jans G, Galjaard S, Corcoy R et al. Effect of Physical Activity and/or Healthy Eating on GDM Risk: The DALI Lifestyle Study. The Journal of Clinical Endocrinology \& Metabolism 2017; 102: 903-913.

15 Barakat R, Pelaez M, Cordero Y, Perales M, Lopez C, Coteron J et al. Exercise during pregnancy protects against hypertension and macrosomia: randomized clinical trial. $\mathrm{Am}$ J Obstet Gynecol 2016; 214: 649.e1-8.

16 Wang C, Wei Y, Zhang X, Zhang Y, Xu Q, Sun Y et al. A randomized clinical trial of exercise during pregnancy to prevent gestational diabetes mellitus and improve pregnancy outcome in overweight and obese pregnant women. Am J Obstet Gynecol 2017; 216: 340-351.

17 Song C, Li J, Leng J, Ma RC, Yang X. Lifestyle intervention can reduce the risk of gestational diabetes: a meta-analysis of randomized controlled trials. Obes Rev 2016; 17: 960-969.

18 Tuomilehto J, Lindström J, Eriksson JG, Valle TT, Hämäläinen H, Ilanne-Parikka P et $a l$. Prevention of type 2 diabetes mellitus by changes in lifestyle among subjects with impaired glucose tolerance. N Engl J Med 2001; 344: 1343-1350.

19 Knowler WC, Barret-Connor E, Fowler SE, Hamman RF, Lachin JM, Walker EA et al. Reduction in the Incidence of Type 2 Diabetes with Lifestyle Intervention or Metformin. N Engl J Med 2002; 346: 393-403.

20 Rönö K, Stach-Lempinen B, Klemetti MM, Kaaja RJ, Pöyhönen-Alho M, Eriksson JG et al. Prevention of gestational diabetes through lifestyle intervention: study design and methods of a Finnish randomized controlled multicenter trial (RADIEL). BMC Pregnancy Childbirth 2014; 14: 70.

21 Koivusalo SB, Rönö K, Klemetti MM, Roine RP, Lindström J, Erkkola M et al. Gestational Diabetes Mellitus Can Be Prevented by Lifestyle Intervention: The Finnish Gestational Diabetes Prevention Study (RADIEL) A Randomized Controlled Trial. Diabetes Care 2016; 39: 24-30.

22 Becker W, Lyhne N, Pedersen A, Aro A, Fogelholm M, Phórsdottir I et al. Nordic Nutrition Recommendations 2004 - integrating nutrition and physical activity. Scandinavian Journal of Nutrition 2004; 48: 178-187.

23 ACOG Committee on Obstetric Practice. ACOG committee opinion. Exercise during pregnancy and the postpartum period. Number 267, January 2002. American College of Obstetricians and Gynecologists. International Journal of Gynecology \& Obstetrics 2002; 77: 79-81.

24 Working group set up by the Finnish Medical Society Duodecim, the Medical Advisory Board of the Finnish Diabetes Association and the Finnish Gynecological Association. 
Gestational Diabetes, Current Care Guidelines. www.käypähoito.fi. 2013; : 1-19.

25 American Diabetes Association. Standards of medical care in diabetes--2008. Diabetes Care. 2008; 31 Suppl 1: S12-54.

26 Meinilä J, Valkama A, Koivusalo SB, Stach-Lempinen B, Lindström J, Kautiainen H et al. Healthy Food Intake Index (HFII) - Validity and reproducibility in a gestationaldiabetes-risk population. BMC Public Health 2016; 16: 680.

27 Ainsworth BE, Haskell WL, Herrmann SD, Meckes N, Basset DR Jr., Tudor-Locke C et al. 2011 Compendium of Physical Activities. Medicine \& Science in Sports \& Exercise 2011; 43: 1575-1581.

28 ACOG Committee on Obstetric Practice. Practice bulletin\# 33: diagnosis and management of preeclampsia and eclampsia. Obstetrics \& Gynecology 2002; 99: 159167.

29 Pihkala J, Hakala T, Voutilainen P, Raivio K. [Characteristic of recent fetal growth curves in Finland]. Duodecim 1989; 105: 1540-1546.

30 Walsh JM, McGowan CA, Mahony R, Foley ME, McAuliffe FM. Low glycaemic index diet in pregnancy to prevent macrosomia (ROLO study): randomised control trial. $B M J$ 2012; 345: e5605.

31 Valkama A, Koivusalo SB, Lindström J, Meinilä J, Kautiainen H, Stach-Lempinen B et $a l$. The effect of dietary counselling on food intakes in pregnant women at risk for gestational diabetes: a secondary analysis of a randomised controlled trial RADIEL. European Journal of Clinical Nutrition 2015; 70: 912-917. 